\title{
GENERACIÓN DE ACIDEZ POR OXIDACIÓN DE PIRITA EN SUELOS SULFATADOS ÁCIDOS INTERIORES DE CLIMA CÁLIDO
}

\section{GENERATION OF ACIDITY BY PYRIT OXIDATION IN INLAND ACID SULPHATE SOILS OF WARM CLIMATE}

\author{
Enrique M. Combatt ${ }^{1}$; Zoraya Y. Martínez ${ }^{2}$ y Manuel S. Palencia ${ }^{3}$ \\ Recibido para evaluación: Septiembre 5 de 2007 - Aceptado para publicación: Mayo 15 de 2008
}

\begin{abstract}
RESUMEN
La presente investigación cuantificó la cantidad de acidez actual total (AAT) y la acidez potencial total (APT) ocasionada en los suelos sulfatados ácidos del bajo Sinú. Aunque se conoce de la manifestación de estos suelos, no se tiene información cuantitativa que permita establecer el impacto de la acidificación por sulfuros sobre los humedales aledaños. Se evaluó un transecto entre los municipios de San Carlos y Cotorra, se seleccionaron muestras de suelo de los horizontes encontrados para ocho sitios diferentes. Las técnicas utilizadas fueron las expuestas por ASSMAC, según la metodología POSA y TSA. Las variables evaluadas fueron AAT y APT en cada uno de los horizontes de los perfiles. Los resultados indican que el azufre se encuentra en concentraciones de 202 a $4800 \mathrm{mg} \mathrm{kg}^{-1}$. AAT en el sitio El Deseo (Municipio de Ciénaga de Oro) presentó valores entre 112 y 604 $\mathrm{kg} \mathrm{ha}^{-1} \mathrm{de}^{+}$y fue nula en los sitios Sabanal y Besito Volao (Municipio de Cotorra). APT sobre suelos sulfatados ácidos potenciales del sector, puede generar aproximadamente $99.745,7 \mathrm{~kg} \mathrm{ha}^{-1} \mathrm{de}_{2} \mathrm{SO}_{4}$ y $45.689 \mathrm{~kg} \mathrm{ha}^{-1}$ de $\mathrm{H}_{2} \mathrm{SO}_{4}$ en suelos sulfatados ácidos actuales; valores bajos se presentaron en la Caimanera (Corregimiento de Carrillo) con 11.890 y $3.528 \mathrm{~kg} \mathrm{ha}^{-1}$ de $\mathrm{H}_{2} \mathrm{SO}_{4}$. Los resultados sugieren que la generación de ácido por la oxidación de sulfuros es un hecho y en algunos sectores existen ingentes cantidades por desarrollarse, ocasionando como consecuencia, grandes impactos negativos en los ecosistemas cenagosos del bajo Sinú.
\end{abstract}

Palabras clave. Suelos sulfatados ácidos, acidez actual, acidez potencial, pirita, oxidación.

\begin{abstract}
The study was done in acid sulphate soils in the Mid and Low Sinu river valley (Cordoba). There is not quantitative information that allows knowing the environmental impact of acidification from pyritic exposure on wetlands. A transect between San Carlos and Cotorra municipalities was established and eight sites were selected. The ASSMAC in Acid Sulphate Soils Analytical Methods, by POSA and TSA technical methodologies were used. The variables were TAA and TPA for each one of the soils horizons. The results

1Universidad de Córdoba, Departamento de Agronómica y Desarrollo Rural. Carrera 6 No. 76 -103 Tel (4) 790 6451 Fax (4) 786 0255. Email: ecombatt@sinu.unicordoba.edu.co

${ }^{2}$ Universidad de Córdoba, Departamento de Geografía.

${ }^{3}$ Químico, mpalencia23@yahoo.com
\end{abstract}


showed that sulfur is between 202 to $4800 \mathrm{mg} \mathrm{kg}^{-1}$. TAA was 112 y $604 \mathrm{Kg} \mathrm{ha}^{-1} \mathrm{of} \mathrm{H}^{+}$in El Deseo while El Sabanal and Besito Volao did not show any amount. APT on potential acid sulphate soils can generate around $99.745,7 \mathrm{Kg} \mathrm{ha}^{-1}$ of $\mathrm{H}_{2} \mathrm{SO}_{4}$ and $45.689 \mathrm{Kg} \mathrm{ha}^{-1}$ of $\mathrm{H}_{2} \mathrm{SO}_{4}$ in actual acid sulfate soils. The lowest amounts were observed in La Caimanera with 11.890 and $3.528 \mathrm{Kg} \mathrm{ha}^{-1} \mathrm{H}_{2} \mathrm{SO}_{4}$. The results suggest that acidity generated from pyritic oxidation may cause significant impact on wetlands of the Sinú river valley.

Keywords. Acid sulphate soils, total actual acidity, total potential acidity.

\section{INTRODUCCIÓN}

En Colombia se conoce la existencia de suelos sulfatados ácidos (SSA) en los departamentos de Córdoba, Valle del Cauca, Bolívar, Atlántico y Boyacá (Combatt, 2004). Los SSA son suelos problemáticos por la dificultad de su diagnóstico en campo, y porque su estudio está asociado con zonas de difícil acceso, como área inundadas, pantanos y ciénagas (Andriesse, 1993; Lin y Lin, 1996). Los problemas inherentes a la acidificación producida por la oxidación del material pirítico, junto con sus consecuentes efectos negativos en el medio ambiente, van desde la disminución del $\mathrm{pH}$, incremento de los contenidos de hierro y aluminio en aguas, la susceptibilidad de peces a la depredación microbiana por deterioro de los tejidos membranosos y la proliferación de cianobacterias y flagelados peligrosos (Willett et al., 1992; Demas et al., 2004).

Esencialmente se pueden definir dos tipos de acidez en los suelos, la acidez mineral y la acidez mineral potencial total. La acidez actual total, análoga a la acidez mineral definida por Konsten et al. (1988), corresponde a la cantidad de acidez del suelo que se encuentra libre; esta comprende la acidez soluble, la acidez intercambiable, la acidez de partículas cargadas variablemente, y la acidez transportada por compuestos sulfatados básicos. En suelos sulfatados ácidos, el mayor ácido mineral es el ácido sulfúrico que se genera por oxidación de los sulfuros (pirita) y el cual es cuantificable por el método de la acidez actual total (McElnea et al., 2002). Existen diferentes métodos para la identificación de SSA a nivel de laboratorio: métodos cuantitativos, semicuantitativos y cualitativos; en los cuales deben considerarse los factores propios de cada sitio, debido a que los resultados no siempre presentan datos consistentes y de fácil interpretación (Janssen y Hendro, 1990). Son diversos los trabajos reportados con este fin, realizados mediante técnicas de oxidación con peróxido, como los reportados por Macdonald et al. (2004), quienes encontraron contenidos de acidez potencial superiores a 100 ton $\mathrm{ha}^{-1} \mathrm{de}$ $\mathrm{H}_{2} \mathrm{SO}_{4^{\prime}}$ en suelos sulfatados ácidos de apariencia uniforme de las riberas del río Tweed (Australia), pero con variaciones considerables tanto en la cantidad de acidez existente como en el contenido de pirita y en la cantidad de especies iónicas solubles e intercambiables. Esto indica que el potencial de producción de acidez en los SSA es muy variado, siendo muchas veces mayor a la acidez actual titulable (AAT) (Jianwu y Shiming, 2002); además, su dinámica depende de factores que pueden operar a diferentes niveles de tiempo y mecanismos de acidificación. Cook et al. (2004) encontraron que la diferencia de las fluctuaciones de ácido durante el periodo de muestreo y en promedio de la velocidad de oxidación in situ fue de 11.5 ton ha-1 de $\mathrm{H}_{2} \mathrm{SO}_{4}$; esto se considera considerablemente mayor que la velocidad de exportación (salida) de la acidez en estos sitios y puede explicar la enorme acidez actual almacenada en dichos suelos. Por lo anterior, el objetivo del presente estudio fue evaluar la cantidad de ácido existente en los SSA actuales y potenciales en el medio y bajo Sinú (Córdoba), y el que potencialmente puede ser generada por la oxidación del material pirítico. 


\section{MATERIALES Y MÉTODOS}

La investigación se realizó en el transecto San Carlos - Cotorra en el Medio y Bajo Sinú (Departamento de Córdoba). A lo largo de esta zona, se llevó a cabo una clasificación fisiográfica con la metodología y clasificación propuesta por Villota (1992). Se ubicaron ocho sitios de muestreos identificados como La Caimanera, El Deseo, Villa Lira y San Carlos, correspondientes a SSA actuales, y La Campiña, El Sabanal, Besito Volao y Bugre correspondientes a SSA potenciales. En cada uno de los sitios se excavaron calicatas de $1 \mathrm{~m} \times 1 \mathrm{~m}$ $\mathrm{x} 1.2 \mathrm{~m}$ de profundidad y fueron colectadas muestras de suelo de $1 \mathrm{~kg}$ en todos los horizontes. Las muestras se almacenaron en bolsas de polipropileno para minimizar su oxidación y se transportaron a las instalaciones del laboratorio en un tiempo inferior a 24 horas, siendo guardadas bajo refrigeración. Para minimizar la oxidación en el laboratorio, se trituraron manualmente, aún en condiciones de humedad de campo, y se secaron a una temperatura de $80{ }^{\circ} \mathrm{C}$ en una estufa. La homogenización de las muestras se realizó mediante trituración en caliente en un molino eléctrico, con un posterior tamizado por una malla de $2 \mathrm{~mm}$. Seguidamente fueron almacenadas bajo refrigeración en recipientes plásticos sellados hasta el momento de su análisis; la determinación de la acidez actual titulable (AAT) y la acidez potencial titulable (APT) se realizaron de acuerdo con la metodología del DNRMI (2004).

\section{Acidez sulfúrica oxidable con peróxido (POSA)}

Se determinó de manera indirecta, correspondiendo a la cantidad de acidez que se puede generar por la oxidación de la pirita presente. Se determinó el azufre pirítico mediante la diferencia entre el presente de manera soluble, en forma de sulfato en la muestra original y el que se encuentra soluble después de la oxidación con peróxido de hidrógeno al $37 \%$. Para la determinación del sulfato soluble,
$25 \mathrm{ml}$ de agua destilada fueron agregados a cinco g de suelo y se centrifugaron a 3000 rpm durante 10 minutos, se filtró y cuantificó el sulfato por espectroscopía de absorción molecular en suspensiones de sulfato de bario a una longitud de onda de $420 \mathrm{~nm}$. Para la determinación del sulfato total generado por la oxidación con peróxido, se tomaron cinco gramos de suelo y se le adicionaron $25 \mathrm{ml}$ de $\mathrm{KCl} 1 \%$, se le adicionaron $5 \mathrm{ml}$ de peroxido de hidrógeno al $30 \%$ con agitación intermitente hasta no observar efervescencia. El sistema se concentró por calentamiento hasta volver a la masa aproximadamente inicial y se filtró. Para eliminar la coloración producida por la materia orgánica se procedió a tomar una alícuota de filtrado y tras la adición de un volumen igual de agua regia y ácido fosfórico, se digestó en un baño de arena. Se transfirió cuantitativamente a matraces de 25 $\mathrm{ml}$ y se cuantificó el sulfato por absorción molecular a suspensiones de sulfato de bario a una longitud de onda de $420 \mathrm{~nm}$. El POSA se determinó mediante la siguiente expresión: POSA $\left(\mathrm{mmol} \mathrm{Kg} \mathrm{suelo}^{-1}\right)=2\left[\mathrm{SO}_{4}\right]_{\mathrm{H} 2 \mathrm{O}}-\left[\mathrm{SO}_{4}\right]_{\mathrm{H} 2 \mathrm{O}}$.

Acidez actual total: Se determinó a partir de cinco gramos de muestra a la cual se le adicionó $50 \mathrm{ml}$ de solución de $\mathrm{KCl} 1.0 \mathrm{~N}$, se agitó por un tiempo de dos horas y se tituló con $\mathrm{NaOH} 0.1 \mathrm{~N}$ hasta alcanzar un $\mathrm{pH}$ estable de 5.5 o superior.

Acidez potencial total: Se determinó mediante la oxidación con $30 \mathrm{ml}$ de peróxido de hidrógeno al $37 \%$ de $5 \mathrm{~g}$ de muestras de suelo previamente tratadas con $50 \mathrm{ml}$ de $\mathrm{KCl} 1.0 \mathrm{~N}$.

La adición del peróxido fue realizada en fracciones de $10 \mathrm{ml}$ en intervalos de tiempo de aproximadamente 30 minutos, seguida de una digestión en un baño de arena entre cada adición del agente oxidante. De manera posterior se adicionó la cantidad de peróxido de hidrógeno necesaria hasta que la reacción cesó. Se tituló con $\mathrm{NaOH} 0.1 \mathrm{~N}$ hasta alcanzar un $\mathrm{pH}$ estable de 5,5 o superior. La titulación debió realizarse mediante el registro continuo de porciones de 
volúmenes adicionados, dejando estabilizar el pH después de cada adición de titulante. El volumen gastado se determinó de manera gráfica por interpolación de una curva de $\mathrm{pH}$ contra volumen de titulante adicionado. Las variables evaluadas fueron la acidez actual total y la acidez potencial total, se analizaron mediante promedios y en forma descriptiva por zona y para cada localidad.

\section{RESULTADOS Y DISCUSIÓN}

\section{Acidez actual titulable en SSA actuales}

En la figura 1, se observa que en Ios SSA actuales existen concentraciones diferenciales de $\mathrm{H}^{+}$que pueden alcanzar aproximadamente los $600 \mathrm{~kg}$ de $\mathrm{H}^{+}$, donde las mayores concentraciones se encuentran en los horizontes diagnósticos y en algunos casos en los horizontes $C$. Se evidencia que en estos sitios existen condiciones químicas adversas por las altas concentraciones $\mathrm{de}^{+}, \mathrm{Al}^{+3}$ y acidez sulfúrica almacenada.

Bennett et al. (2004), informan que los SSA actuales contienen grandes cantidades de acidez sulfúrica, hierro y oxihidróxidos de aluminio, por consiguiente considerables volúmenes de acidez almacenada que ocasionan toxicidad a la fauna y flora. Esta condición se presenta por la intervención humana sobre el medio, mediante prácticas de drenaje y mecanización continua, que oxidan los compuestos de sulfuros y aumentan la acidez del suelo. Cook et al. (2004) establecen que la generación de acidez a partir de la oxidación de la pirita en suelos sulfatados ácidos requiere el transporte de oxígeno a través del perfil. La disminución del oxígeno no solo ocurre por la reacción química con la pirita, sino también por diversos procesos biológicos asociados con la respiración de las plantas y los microorganismos. Demas et al. (2004) encuentran que dos son los mecanismos responsables de la producción de ácido en estos suelos: la oxidación e hidrólisis de hierro móvil y la oxidación de sulfuros presentes en forma de pirita; por lo tanto, estas áreas representan una limitación al desarrollo agrícola, por la gran cantidad de ácido que interfiere en el desarrollo de los cultivos, necesitándose una gran inversión económica para su adecuación y explotación.

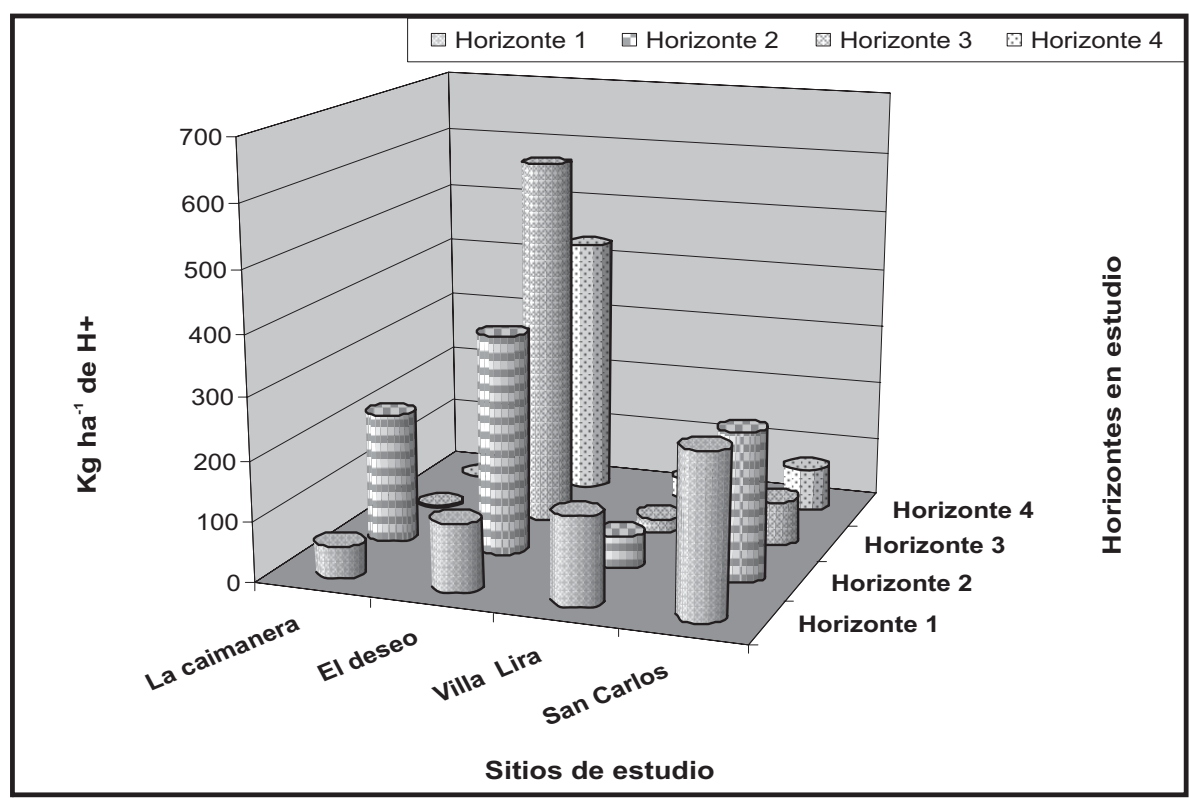

Figura 1. Acidez actual en suelos sulfatados ácidos actuales en Córdoba 
Acidez actual titulable en SSA potenciales

En la figura 2 se observa que la producción de $\mathrm{H}^{+}$para los sitios estudiados es menor que en los suelos actuales, con valores de aproximadamente $100 \mathrm{~kg} \mathrm{ha}^{-1} \mathrm{de} \mathrm{H}^{+}$, lo que está directamente relacionado con la permanencia de estas zonas bajo un estado reductor, impidiendo la oxidación de la pirita y la posible formación de ácido sulfúrico, procesos causantes de una mayor acidificación. Estos resultados concuerdan con Konsten y Brinkman (1984), quienes trabajando en Typic Sulfaquent encontraron valores cercanos a $128 \mathrm{~kg} \mathrm{ha}^{-1} \mathrm{de}^{+}$en un suelo sulfatado ácido potencial. Otra condición encontrada, es que hay sitios de muestreo donde no se determinó acidez actual, por la alta acumulación de sales y alto contenido de humedad, lo cual no permitió que se potencializara la producción de $\mathrm{H}^{+}$, manteniendo la reacción del suelo con $\mathrm{pH}$ mayor a 5.5. Lo anterior explica, que si estos sitios son drenados existe una alta probabilidad de no ser aptos para la producción de cultivos.

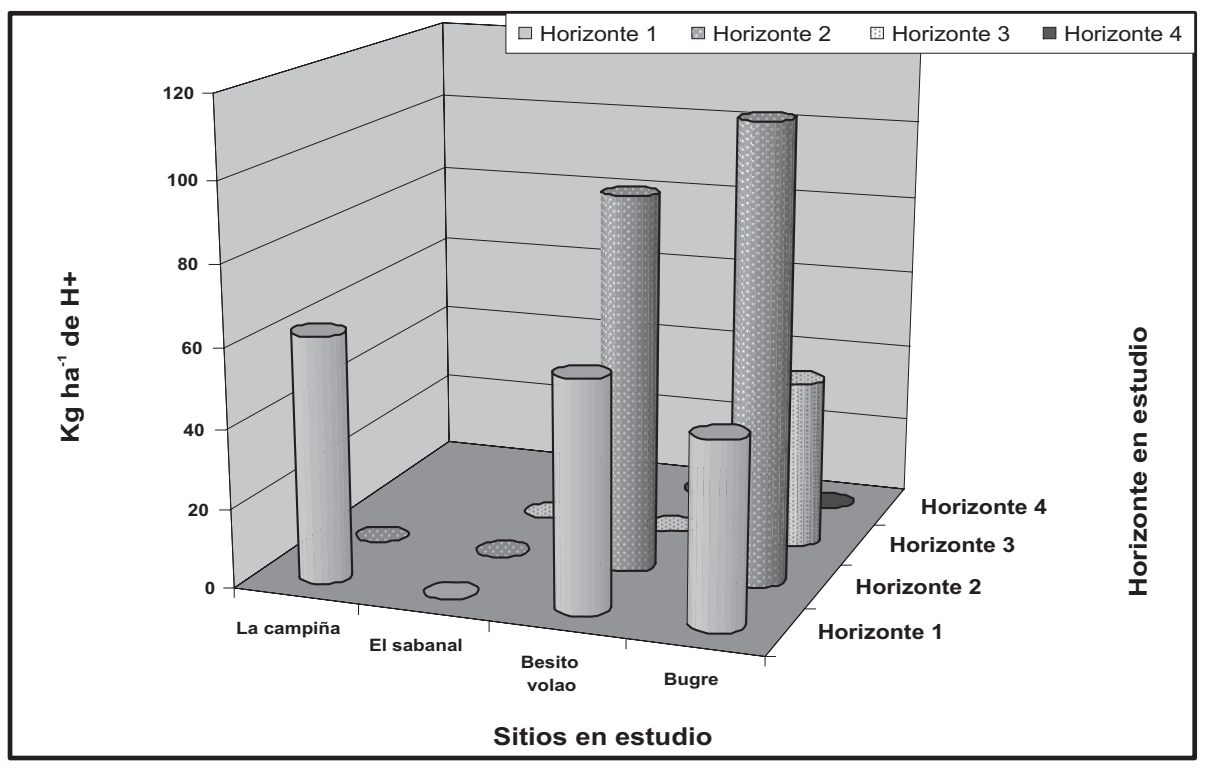

Figura 2. Acidez Potencial Total en suelos sulfatados ácidos potenciales en el Medio y Bajo Sinu - Córdoba

La acidez potencial que se puede generar en estos suelos puede alcanzar valores de hasta $100.000 \mathrm{~kg} \mathrm{ha}^{-1}$ de $\mathrm{H}_{2} \mathrm{SO}_{4}$ (Figura 3), en aquellos suelos que hoy día hacen parte de la Ciénaga Grande del Bajo Sinú, encontrándose los máximos valores en los endopedones, donde se presenta el horizonte jarosítico, en subórdenes de suelos clasificados como Typic Sulfaquent y Typic Sulfaquept; aquí la mayor acidificación a generarse se ocasionaría en los Entisoles, los cuales tienen concentraciones de azufre de aproximadamente $4800 \mathrm{mg} \mathrm{kg}^{-1}$. Con respecto a los horizontes subyacentes de SSA actuales, se observa que existe la posibilidad de producir altas cantidades de acidez potencial, en caso de ser expuestos a la oxidación. Similares resultados fueron encontrados por Macdonald et al. (2004) quienes identificaron contenidos de acidez potencial superiores a $100.000 \mathrm{~kg} \mathrm{ha}^{-1}$ de $\mathrm{H}_{2} \mathrm{SO}_{4}$ en suelos sulfatados ácidos de apariencia uniforme de las riveras del río Tweed (Australia), y Rayment (2002) quien encontró niveles medios de AAT a $1.5 \mathrm{~m}$, entre $1.04 \mathrm{~kg} \mathrm{t}^{-1} \mathrm{de}_{2} \mathrm{SO}_{4}$ y $2.96 \mathrm{~kg} \mathrm{t}^{-1}$ de $\mathrm{H}_{2} \mathrm{SO}_{4} \mathrm{y}$ niveles medios de APT de $9.24 \mathrm{~kg} \mathrm{t}^{-1} \mathrm{de}_{2} \mathrm{SO}_{4^{\prime}}$ el porcentaje de APT que se incrementa con la profundidad del perfil, se puede explicar por la considerable acidez potencial almacenada en estos suelos de origen costeros. 


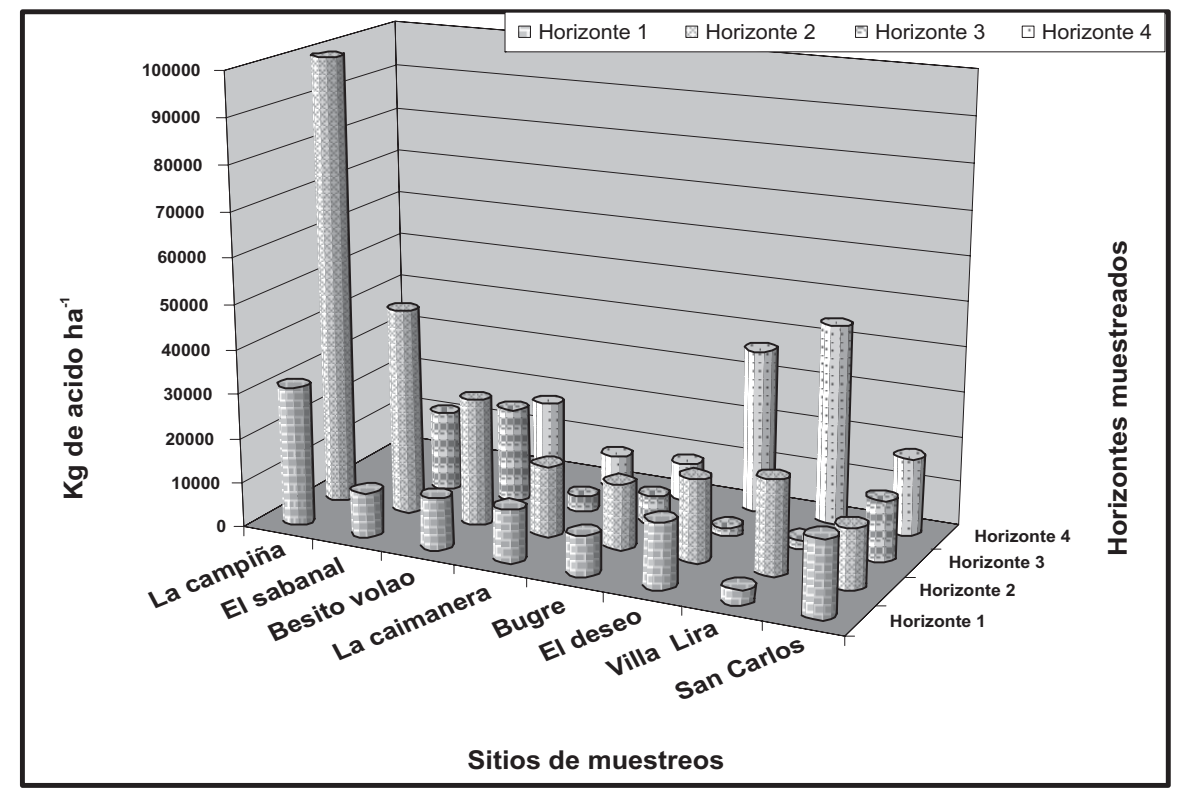

Figura 3. Acidez potencial total que se puede generar en suelos sulfatados ácidos actuales y potenciales en el medio y bajo Sinú Córdoba

En la Tabla 1, se presentan algunas conversiones establecidas mediante la determinación del azufre oxidable en esta investigación, para los suelos sulfatados ácidos del sector en estudio en el Departamento de Córdoba. Las unidades usadas expresan resultados analíticos basadas en 1 mol de pirita produciendo 2 moles de ácido sulfúrico o 4

Tabla 1. Conversiones para algunas unidades reportadas en análisis de suelos sulfatados ácidos en Córdoba

\begin{tabular}{|c|c|c|c|c|c|}
\hline \% Sox* & $\begin{array}{l}\text { moles } \mathrm{H}^{+} \mathbf{k g}^{-1} \\
(\mathrm{~S} \% \mathrm{x} 0,624)\end{array}$ & $\begin{array}{l}\text { moles } \mathrm{H}^{+} \mathrm{t}^{-1} \\
(\mathrm{~S} \% \mathrm{x} \quad 624)\end{array}$ & $\begin{array}{l}\mathrm{Kg} \mathrm{H}_{2} \mathrm{SO}_{4} \mathbf{t}^{-1} \\
(\mathrm{~S} \% \times \mathbf{3 0}, 59)\end{array}$ & $\begin{array}{l}\mathrm{Kg} \mathrm{cal} \mathrm{t}^{-1} \text { suelo } \\
\text { (Factor } 1,5)\end{array}$ & ton cal ha-1 \\
\hline 0.021 & 0.013 & 13.03 & 0.639 & 0.958 & 11.33 \\
\hline 0.026 & 0.016 & 15.93 & 0.781 & 1.172 & 6.52 \\
\hline 0.032 & 0.020 & 20.18 & 0.989 & 1.484 & 5.29 \\
\hline 0.047 & 0.029 & 29.27 & 1.435 & 2.152 & 26.01 \\
\hline 0.067 & 0.042 & 41.73 & 2.046 & 3.069 & 8.01 \\
\hline 0.069 & 0.043 & 42.94 & 2.105 & 3.157 & 11.80 \\
\hline 0.086 & 0.054 & 53.70 & 2.632 & 3.949 & 20.13 \\
\hline 0.106 & 0.066 & 66.45 & 3.257 & 4.886 & 23.77 \\
\hline 0.109 & 0.068 & 68.26 & 3.346 & 5.020 & 20.40 \\
\hline 0.124 & 0.078 & 77.57 & 3.803 & 5.704 & 55.02 \\
\hline 0.131 & 0.082 & 81.65 & 4.002 & 6.004 & 17.82 \\
\hline 0.148 & 0.092 & 92.42 & 4.531 & 6.796 & 68.46 \\
\hline 0.148 & 0.092 & 92.16 & 4.518 & 6.777 & 27.69 \\
\hline 0.172 & 0.107 & 107.15 & 5.253 & 7.879 & 66.70 \\
\hline 0.178 & 0.111 & 111.07 & 5.445 & 8.168 & 30.90 \\
\hline 0.547 & 0.341 & 341.37 & 16.735 & 25.102 & 22.12 \\
\hline
\end{tabular}

*\%Sox. Azufre oxidable 
moles de $\mathrm{H}^{+}$, con su equivalente en encalado usando un factor de seguridad de 1.5. Los datos obtenidos son similares a los establecidos por el DNRMI (2004), para suelos de pantano y zonas de manglares en las costas de Australia, con la diferencia de que el \%Sox para los suelos interiores de Córdoba no sobrepasan el valor de $0,6 \%$, mientras que en los suelos costeros de Australia se han encontradas concentraciones de 5\%; para el caso australiano, el azufre oxidable puede ser neutralizado por 234 ton de cal agrícola, y en Córdoba se requerirían aproximadamente 23 ton para neutralizar la acidez generada en el primer horizonte.

\section{CONCLUSIONES}

- La acidificación por sulfuros en suelos (sulfatados ácidos) de Córdoba está ocurriendo y seguirá manifestándose inevitablemente, si siguen siendo drenados los humedales y sus áreas aledañas.
- Las altas cantidades de ácido sulfúrico que se pueden generar en estas zonas aledañas a sistemas cenagosos afectarían el medio ambiente.

- Los suelos sulfatados ácidos potenciales tienen una alta reserva de acidez que en la actualidad no se manifiesta por la continua reducción en que se mantienen estas áreas, debido a la temporada anual de lluvias.

- El porcentaje de azufre oxidable (\%Sox) que existe en los suelos sulfatados ácidos del bajo Sinú en Córdoba analizados en el presente estudio, oscila entre 0.021 y 0.547 , y pueden ser neutralizados con 23 ton de cal aproximadamente.

- Se recomienda implementar otras metodologías para medición de acidez actual y potencial que permitan correlacionar los resultados obtenidos en suelos sulfatados ácidos interiores tropicales, con las de otras condiciones ambientales.

\section{BIBLIOGRAFÍA}

Andriesse, W. 1993. Acid sulphate soils: Diagnosing the illness. En: Dent D. y Mensvoort M. (Ed). Proceedings of The Symposium on Acid Sulphate Soils, Ho Chi Minh City, 53:11-30

Bennett, C.; White, I.; Keene, A.; Melville, M. y Reynolds, J. 2004. Super Soil. Australian New Zealand Soils Conference, University of Sydney, CDROM

Combatt, E. 2004. Efecto del encalamiento y el lavado sobre algunas propiedades químicas de un suelo sulfatado ácido magnésico del valle del rió Sinú.
Tesis M. Sc., Universidad Nacional de Colombia, Palmira

Cook, F.; Dobos, S.; Carlin G. y Millar G. 2004. Oxidation rate of pyrite in acid sulfate soils: in situ measurements and modeling. Australian Journal of Soil Research 42(6):499-507

Demas, A.; Hall, A.; Fanning, B.; Rabenhorst, B. y Dzantor, B. 2004. Acid sulfate soils in dredged materials from tidal Pocomoke Sound in Somerset County, MD, USA. Australian Journal of Soil Research 42(6):537-545 
DNRMI (Department of Natural Resources, Mines, Indoorroopilly). 2004. Acid Sulfate Soils Laboratory Methods Guidelines, Quesland, 132p.

Janssen, J. y Hendro, P. 1990. Acid sulphate soils; field characteristics and mapping. Papers Workshop on Acid Sulphate Soils in the Humid Tropics. International Institute for Land Reclamation and Improvement, p5161

Jianwu, W. y Shiming, L. 2002. Sulfur and its acidity in acid sulfate soil of Taishan coastal plain in southern China. Communications in Soil Science and Plant Analysis 33:(3-4):579-593

Konsten, C. y Brinkman, R. 1984. A field laboratory method to determine total potential and actual acidity in acid sulphate soils. Proceedings Symposium on Acid Sulphate Soils. Dakar, p106

Konsten, C; Brinkman, R. y Andriesse W. 1988. A field laboratory method to determine total potencial and actual acidity in acid sulphate soils. Proceedings Symposium on Acid Sulphate Soils. Dakar, p106-134
Lin, C. y Lin, C. 1996. Human influences on the development of acid sulphate soils in the pearl River (The Zhujiang) delta. Pedosphere 6(1):1-10

Macdonald, B; White, I.; Keene, A.; Melville.; M. y Reynolds, J. 2004. Acidity, metals and acid sulfate soils. 3rd Conference SuperSoil Australian New Zealand Soils.

McElnea, A.; Ahern, C. y Menzies, N. 2002. The measurement of actual acidity in acid sulfate soils and the determination of sulfidic acidity in suspension after peroxide oxidation. Australian Journal of Soil Research 40:1133-1157

Rayment, G. 2002. Actual and potential pyritic acidity of new canelands. Communications in Soil Science and Plant Analysis 33(15-18):3033-3044

Villota, H. 1992. El sistema CIAF de clasificación fisiográfica del terreno. CIAF - IGAC, Bogotá, 13(1):55-70

Willett, I.; Melville, M. y White I. 1992. Acid drainwaters from potencial sulhate soils and their impact on estuarine ecosystems. Proceedings of Simposium on Acid Sulphate Soils, Ho Chi Minh City, 1:419-425 